\section{P015 INTRODUCTION OF CHLAMYDIA AND GONORRHEA OPT- OUT TESTING IN A SHORT-TERM CORRECTIONAL FACILITY IN ALBERTA, CANADA}

'Jennifer Gratrix, ${ }^{2}$ Diane Payne, ${ }^{1}$ Petra Smyczek*, 'Lynn Eagle, ${ }^{2}$ Keith Courtney, ${ }^{3}$ Rabia Ahmed. ${ }^{1}$ Alberta Health Services, STI Services, Edmonton, Canada; ${ }^{2}$ Alberta Health Services, Corrections Health, Edmonton, Canada; ${ }^{3}$ University of Alberta, Medicine, Edmonton, Canada

\subsection{6/sextrans-2019-sti.224}

Background Incarceration provides an opportunity for screening and treatment of STBBI in high-risk groups. The purpose of this study was to evaluate the uptake and outcomes of optout screening at time of admission.

Methods Between March and September 2018, all individuals $\leq 30$ years admitted to a short-term correctional facility in Alberta, Canada were offered urine nucleic acid amplification test (Aptima Combo $2^{\circledR}$, Hologic Inc., Marlborough, MA) for Chlamydia trachomatis (CT) and Neisseria gonorrhea (NG), upon admission. Admission line-lists recorded offering of optout testing and reasons for testing non-completion. Laboratory data extracts provided test results by matching collection date with unique patient identifiers. Simple descriptive statistics analysis were completed.

Results A total of 1,735 (1,295 males) admissions were recorded, with a median age of 23 years (IQR: 21-25). Most $(92.7 \% ; n=1,608)$ of individuals were offered opt-out testing; reasons for not offering testing included inability to consent $(35.1 \% ; n=39)$, operational limitations $(28.8 \% ; n=32)$, guardianship issues $(10.8 \% ; n=12)$, other concerns $(9.0 \% ; n=10)$, and missing $(16.2 \% ; n=18)$. Of those offered testing, $32.3 \%$ $(n=520)$ consented. Reasons for not consenting included: no perceived risk $(35.3 \% ; \mathrm{n}=384)$, recently tested $(16.6 \%$; $\mathrm{n}=181)$, deferred testing $(13.3 \% ; \mathrm{n}=145)$, declined $(11.0$; $\mathrm{n}=120)$, not sexually active $(4.7 \% ; \mathrm{n}=51)$, other $(5.9 \%$; $\mathrm{n}=65)$, and missing $(13.1 \% ; \mathrm{n}=142)$. The positivity rate for CT was $18.0 \%(n=83)$ and NG was $(12.6 \% ; n=58)$. Women were more likely to test positive for CT $(26.1 \%$ vs $15.2 \%$, $\mathrm{P}=0.008)$ and $\mathrm{NG}(21.8 \%$ vs $9.3 \%, \mathrm{P}<0.001)$ than men.

Conclusion Opt-out testing at admission proved to be feasible, although uptake was relatively low, CT and NG positivity rates were high. With the majority of incarcerated individuals in Canada being held in short-term correctional facilities, intensification of screening strategies to an opt-out model is effective for a large number of high-risk individuals.

Disclosure No significant relationships.

\section{P016 KNOWLEDGE AND TESTING OF HIV AMONG MEN AND WOMEN IN INDIA: EXPLORING TEST AND TREAT MODEL OF HIV PREVENTION}

Santosh Sharma*. International Institute for Population Sciences, Population Studies, Mumbai, India

\subsection{6/sextrans-2019-sti.225}

Background The government of India adopted the Test and Treat model of human immunodeficiency virus (HIV) prevention. This study has made an attempt to explore the effectiveness of the Test and Treat approaches by analyzing the knowledge and testing of HIV among male and female.

Methods The study has used last two rounds of National Family Health Survey data, providing community-based insights in the testing of HIV. Descriptive and multivariate techniques have been used to analyze the nationally representative sample of 69,751 men and 124,385 women in 2005-06 and 103,411 men and 121,118 women age $15-49$ in 201516 respectively.

Results Women, who are educated, residing in urban areas, and from better economically households are more prone to have knowledge of place of HIV testing and also have ever undergone HIV testing than their men counterparts. It is a welcome change given the existing HIV scenario, with a continuously narrowing gender gap in new HIV infections. Women and men having positive attitude towards stigma and discrimination to people living with HIV (PLHIV) are in better agreement to ever being tested. Further, women and men having knowledge about antiretroviral therapy (ART) are 1.6 times $(\mathrm{p}<0.001)$ and 1.8 times $(\mathrm{p}<0.001)$ more likely to have ever been tested for HIV. The 'test and treat' model of HIV in India portrays a women-centric effort to ensure HIV testing as part of their ANC, resulting in a substantial increase in ever being tested.

Conclusion This study concluded that HIV testing and treatment has improved considerably over the last decade among women and men. Despite an enhancement in coverage of HIV testing, the existing disparities in HIV testing and treatment require policy instruments with an integrated approach. Government should work in close collaboration with communities/ key stakeholders, and efficiently use their resources to provide evidence-based HIV prevention and treatment interventions. Disclosure No significant relationships.

\section{P017 EFFECT OF TIME CHANGE ON ADOLESCENT AND YOUNG PEOPLE ACCESSIBILITY TO AYFHC IN AKURE, ONDO STATE NIGERIA}

Onesimus Aiwanfo*. Kids and Teens Resource Centre, Monitoring and Evaluation Learning and Reporting, Akure, Nigeria

\subsection{6/sextrans-2019-sti.226}

Background Government in Ondo state have made significant effort in establishing youth friendly health care centres to increase young people's access to health and social services. By it became very frustrating to see very few young people patronizing such centres due $8 \mathrm{am}-4 \mathrm{pm}$ working hours. Change in time to $4 \mathrm{pm}-8 \mathrm{pm}$ showed a significant difference in young people access to AYFHC services.

Methods Using Participatory Learning in action and community engagement We discovered young people 10-35 years in Akure either attend school from $8 \mathrm{am}-4 \mathrm{pm}$ or go to centres where they skills from $8 \mathrm{am}-6 \mathrm{pm}$. With this kind of schedule, it became very difficult for young people to leave school or their skill centres for AYFHC services. In 2017, there was an extension in service time to $8 \mathrm{pm}$, there was a massive increase of young people accessing social and health services at this centre's from $4 \mathrm{pm}-8 \mathrm{pm}$ from 2 persons per day to 12 persons per day.

Results It was observed that the additional 10 persons came between $4 \mathrm{pm}$ to $8 \mathrm{pm}$. Most of the young people preferred the evening hours because it was very convenient and it allows them do their daily activities. Also it reduced stigmatization because absence at school or work leads to questions been raised.

Conclusion Provision of AYFHC isn't enough, passionate Friendly health workers should be employed and Service time 
should be convenient for young people to come as well as the location should be strategic.

Disclosure No significant relationships.

\section{P018 PAY-IT-FORWARD GONORRHEA AND CHLAMYDIA TESTING AMONG CHINESE MEN WHO HAVE SEX WITH MEN: A CLUSTER RANDOMIZED CONTROLLED TRIAL}

\begin{abstract}
${ }^{1}$ Tiange Zhang*, ${ }^{2}$ Fan Yang, ${ }^{3}$ Weiming Tang, ${ }^{3}$ Wenting Huang, ${ }^{1}$ Yehua Wang, ${ }^{4}$ Marcus Alexander, ${ }^{4}$ Laura Forastiere, ${ }^{4}$ Navin Kumar, ${ }^{3}$ Katherine Li, ${ }^{5}$ Fei Zou, ${ }^{6}$ Ligang Yang, ${ }^{7}$ Guodong Mi, ${ }^{3}$ Amy Lee, ${ }^{8}$ Weizan Zhu, ${ }^{9}$ Peter Vickerman, ${ }^{3}$ Dan $W u,{ }^{6}$ Bin Yang, ${ }^{4}$ Nicholas Christakis, ${ }^{3}$ Joseph Tucker. 'University of North Carolina Project-China, Guangzhou, China; ${ }^{2}$ University of North Carolina Project - China, Guangzhou, China; ${ }^{3}$ UNC Project-China, Guangzhou, China; ${ }^{4}$ Yale Institute for Network Science, New Haven, USA; ${ }^{5}$ University of North Carolina at Chapel Hill, Department of Biostatistics, Chapel Hill, USA; ${ }^{6}$ Dermatology Hospital, Southern Medical University, Guangzhou, China; ${ }^{7}$ BlueD, Beijing, China; ${ }^{8}$ Zhitong Guangzhou LGBT Center, Guangzhou, China; ${ }^{9}$ University of Bristol, School of Social and Community Medicine, Bristol, UK
\end{abstract}

\subsection{6/sextrans-2019-sti.227}

Background Gonorrhea and chlamydia testing is poor among Chinese MSM with HIV risk. Furthermore, gonorrhea and chlamydia testing programs are poorly funded and unlinked to HIV testing services. Pay-it-forward offers an individual a gift (e.g. an STD test) and then asks whether they would like to give a gift to another person. This study assesses the effectiveness of a pay-it-forward program in increasing gonorrhea/chlamydia testing among MSM compared to standard of care.

Methods We conducted a cluster randomized controlled trial at three HIV testing sites run by MSM community-based organizations in Beijing and Guangzhou (NCT03741725). We included MSM aged 16 or older who had met indications for testing based on US CDC guidelines. Twenty clusters of 10 men each were randomized into pay-it-forward and standard of care arms. In pay-it-forward, men were offered free testing and then given the option to donate money toward future participants' tests; in standard of care, testing was offered at the standard price of $150 \mathrm{RMB}$ (US\$22). The primary outcome was dual gonorrhea/chlamydia test uptake ascertained by administrative record. Generalized estimating equations were used to assess the population-averaged effect of the pay-it-forward intervention. This analysis focuses on the primary comparison between pay-it-forward and standard of care.

Results Overall, 201 participants were recruited. Most participants were younger than 30 years old $(67.5 \%, 127 / 188)$ and had college or above education (85.1\%, 165/194). 43.1\% (69/ $160)$ reported condomless anal sex in the last three months. Gonorrhea/chlamydia testing rates were $56.4 \%(57 / 101)$ in pay-it-forward and $18.0 \%(18 / 100)$ in standard of care. Gonorrhea/chlamydia testing rates were three times higher in payit-forward compared to standard of care (RR:3.14, p $<0.001$, $95 \% \mathrm{CI}=1.80-5.45)$. $94.7 \%(54 / 57)$ of pay-it-forward participants donated toward future participants, with an average amount of 53.6RMB (US\$8).

Conclusion Findings suggest that pay-it-forward increases gonorrhea/chlamydia testing and may inform the integration of STD and HIV testing services among MSM.

Disclosure No significant relationships.

\section{P019 \\ IMPLEMENTING HOME-SAMPLING STRATEGIES TO ENGAGE YOUNG HETEROSEXUALS IN CHLAMYDIA TRACHOMATIS RE-TESTING AND PEER-TESTING}

Nicole Dukers-Muijrers*, Jeanine Leenen, Christian Hoebe. Public Health Service South Limburg, Maastricht University Medical Center (MUMC), Sexual Health, Infectious Diseases and Environmental Health, Medical Microbiology, Care and Public Health Research Institute (CAPHRI), Heerlen, Netherlands

\subsection{6/sextrans-2019-sti.228}

Background Home-sampling is an increasingly used method to promote testing for Chlamydia trachomatis. Here, we implement and evaluate home-sampling for re-testing and peer-testing in young $(<25$ years) heterosexual men and women.

Methods At our STI clinics (Limburg, Netherlands, 20152018), indexes, i.e. young heterosexuals diagnosed with chlamydia, were (a) offered a self-sampling test for a peer, and (b) offered to receive an SMS-reminder after 5 months to order a re-test for themselves and a peer. Test offers were free-of-charge self-sampling tests for chlamydia and gonorrhea (urine sample for men; vaginal and rectal swabs for women). We interviewed care providers on implementation-barriers and facilitators and performed a process evaluation on test-uptake and overall (genital or rectal) STI positivity.

Results Of 1709 indexes (1221 women): (a) 571 (33.4\%) were given a test for a peer; 211 (37.0\%) peers tested and peer-positivity was $18.5 \%$ (17.9\% in women-including 3 cases of gonorrhea and $12.8 \%$ rectal chlamydia; $19.5 \%$ in men). (b) $1048(61.3 \%)$ accepted to receive a re-test reminder; 417 $(39.8 \%)$ ordered the re-test and $266(63.8 \%)$ re-tested with $13.9 \%$ re-test positivity (15.6\% in women -including $10.4 \%$ rectal chlamydia-, $8.2 \%$ in men - including one gonorrhea case). $155 / 417(37.2 \%)$ also ordered a peer-test; $62(40 \%)$ peers tested but only $6.5 \%(n=4)$ were positive. In (a) and (b), nearly half of positive peers never tested before; rectal infections in women were frequently without a genital infection. Interviews with care-providers revealed problems in implementing logistics which could be solved by an easy-touse online system.

Conclusion A future implementation using an online system needs to improve test-kit returns by including reminders or provisionary payment, and strategies to help indexes find and motivate high risk peers. High STI positivity in peers (many first time testers) from clinic-indexes reveals the need to especially target these peers to tap into a hidden (untested) infected population.

Disclosure No significant relationships.

\section{P020 URISPONGETM FACILITATE URINE COLLECTION AND TRANSPORTATION FOR THE DETECTION OF STD WITH THE ANYPLEXTM II STI-7 ASSAY}

${ }^{1}$ Santina Castriciano*, ${ }^{2}$ Anna Archenti, ${ }^{2}$ Patrizia Biagiola, ${ }^{2}$ Debora Pasquali, ${ }^{2}$ Marina Foti. ${ }^{1}$ Copan Italia Spa, Scientific Affairs, Hamilton, Canada; ${ }^{2}$ 2ATS Città Metropolitana di Milano-Laboratorio di Prevenzione, Milano, Italy

\subsection{6/sextrans-2019-sti.229}

Background Molecular urine collection devices are not supporting bacteria culture. Copan UriSponge ${ }^{\mathrm{TM}}$ consists of a leak-proof screw-cap tube with 3 sponges containing preservative salts attached to a plastic stick to absorb and retain urine during transport and prevent bacterial overgrowth. Urines collected in UriSponge ${ }^{\mathrm{TM}}$ were compared to urine samples 